\title{
Light intensity, temperature, and thickness dependence of the open-circuit voltage in solid-state dye-sensitized solar cells
}

\author{
Henry J. Snaith,* Lukas Schmidt-Mende, and Michael Grätzel \\ Institut de Chimie Physique, École Polytechnique, Fédérale de Lausanne, CH-1015 Lausanne, Switzerland \\ Marco Chiesa \\ Cavendish Laboratory, J. J. Thomson Avenue, Cambridge CB3 OHE, United Kingdom
}

(Received 7 March 2006; published 12 July 2006)

\begin{abstract}
We present an analytical and experimental investigation into the origin of the open-circuit voltage in the solid-state dye-sensitized solar cell. Through Kelvin probe microscopy, we demonstrate that a macroscopically uniform electric field exists throughout the nanocomposite between the electrodes. Considering a balance between drift and diffusion currents, and between charge generation and recombination, we develop an analytical expression for the open-circuit voltage which accurately follows experimental data. We find the opencircuit voltage increases with light intensity as $1.7 \mathrm{kT} / \mathrm{q}$, where $T$ is absolute temperature, however it decreases with increasing temperature and device thickness. The intensity dependence arises from the charge generation rate increasing more strongly with intensity than the recombination rate constant, resulting in increased chemical potential within the device. We find that the temperature dependence arises from a reduction in the charge lifetime and not from increased charge diffusion and mobility. The thickness dependence is found to derive from two factors; first, charge recombination sites are distributed throughout the film, enabling more charges to recombine in thicker films before influencing the potential at the electrodes, and second, the average optical power density within the film reduces with increasing film thickness.
\end{abstract}

DOI: 10.1103/PhysRevB.74.045306

\section{INTRODUCTION}

There is widespread interest in the development of cheap, clean alternatives to current power generation methods due to concerns of the environmental impact of "dirty" fuels. The solid-state dye-sensitized solar cell is one of the most promising candidates to power sustainable growth of the future. It comprises of a nanostructured semiconductor electrode sensitized with dye molecules and infiltrated with a hole transporting organic material. ${ }^{1,2}$ Light absorption, generation, and separation takes place at the dye interface, with subsequent electron and hole transport to the collection electrodes in their respective materials. Most of the development of this system has been undertaken in the chemistry and synthesis of new materials. ${ }^{3-7}$ Although some is understood, ${ }^{8-12}$ much of the operational physics remains a mystery. Understanding the physical mechanisms which govern the device operation is essential for further development. One of the key parameters which governs the attainable power from such a device is the open-circuit voltage $\left(V_{\text {oc }}\right)$, which is the maximum voltage the device can generate. Here, we present a comprehensive experimental investigation of complete solar cells in order to clarify the function of each process occurring within the device. We develop an analytical model which describes the temperature, thickness, and light intensity dependence of the open-circuit voltage. This gives remarkable insight into the physical mechanisms which govern the device operation. The temperature dependence arises from a weakly temperature dependent recombination rate constant which acts as to reduce the open-circuit voltage with increasing temperature. The thickness dependence is multiply due to the average optical power density within the film reducing with increasing thickness, and due to the recombination sites being distributed throughout the film, implying that each charge can
PACS number(s): 84.60.Jt, 72.80.Le, 85.60.- q

contribute less to the voltage measured at the electrodes in thicker films. The increase of open-circuit voltage with intensity is due to the charge generation rate increasing more strongly with intensity than the recombination rate constant, enabling a larger chemical potential to build up within the device.

\section{EXPERIMENTAL}

The dye-sensitized solar cells used in this study were fabricated as follows: Fluorine doped tin oxide (FTO) coated glass sheets $(15 \Omega / \square$, Pilkington) were etched with zinc powder and $\mathrm{HCl}(4 \mathrm{~N})$ to form the required electrode pattern. The sheets were subsequently cleaned with soap $(2 \% \mathrm{Tel}-$ manex in water), distilled water, acetone, ethanol and finally treated under oxygen plasma for 20 minutes to remove any organic residues. The sheets were then coated with a compact layer of $\mathrm{TiO}_{2}(100 \mathrm{~nm})$ by aerosol spray pyrolysis at $450{ }^{\circ} \mathrm{C}$ using oxygen as the carrier gas. ${ }^{13,14} \mathrm{~A}$ homemade $\mathrm{TiO}_{2}$ nanoparticle paste ${ }^{15}$ was doctor-bladed onto the sheets to give a dry film thickness between 1 and $4 \mu \mathrm{m}$, governed by the height of the doctor blade. These sheets were then slowly heated to $500{ }^{\circ} \mathrm{C}$ (ramped over 30 minutes) and baked at this temperature for 30 minutes under an oxygen flow. After cooling, the sheets were cut into slides of the required size and stored in the dark until further use. Prior to fabrication of each set of devices, the nanoporous films were soaked in a $0.02 \mathrm{M}$ aqueous solution of $\mathrm{TiCl}_{4}$ for 6 hours at room temperature in the dark. After rinsing with deionized water and drying in air, the films were baked once more at $500{ }^{\circ} \mathrm{C}$ for 45 minutes under oxygen flow with subsequent cooling to $60{ }^{\circ} \mathrm{C}$ and placed in a dye solution overnight. The ruthenium-based dye used for sensitization was "K51," a bipyridine complex with ion coordinating moieties. ${ }^{16,17}$ 
The dye solution comprised of $300 \mu \mathrm{M}$ of $\mathrm{K} 51$ in acetonitrile and tert-butyl alcohol (volume ratio: 1:1) The hole transporting material used was 2,2',7,7'tetrakis( $N, \quad N$-di-methoxypheny-amine)-9,9'-spirobifluorene (Spiro-MeOTAD), which was dissolved in chlorobenzene (CB) $(180 \mathrm{mg} / \mathrm{mL})$. Tertbutyl pyridine (tbp) was added straight to the solution (1:57 tbp:CB). Lithium TFSI (ionic dopant) and $\mathrm{SbCl}_{4}$ (chemical p-dopant) were separately predissolved in acetonitrile at 170 and $7 \mathrm{mg} / \mathrm{mL}$, respectively. These solutions were then added to the hole-transporter solution at 1:27 vol:vol and 1:31 vol:vol, respectively. The dyed nanoporous films were briefly rinsed in acetonitrile and dried in air for one minute. A small quantity $(70 \mu \mathrm{L})$ of the Spiro-MeOTAD solution was dispensed onto each dyed substrate and left for $40 \mathrm{~s}$ before spin-coating at $2000 \mathrm{rpm}$ in air. The films were then placed in an evaporator where $30 \mathrm{~nm}$ thick gold electrodes were deposited through a shadow mask under high vacuum $\left(10^{-6} \mathrm{mBar}\right)$. The device area was defined as the overlap between the FTO anode and gold cathode and was approximately $0.16 \mathrm{~cm}^{-2}$. Current voltage measurements were performed under a rough vacuum, in the dark and under illumination from a halogen lamp using neutral filters to vary the incident illumination intensity. The Kelvin probe microscope setup used to measure the surface potential of "in-plane" devices under ultrahigh vacuum was identical to that described by Burgi et al. ${ }^{18}$

\section{RESULTS AND DISCUSSION}

The photovoltaic operation of dye-sensitized solar cells does not follow that predicted for conventional $p-n$ junctions. ${ }^{19}$ It is therefore necessary to develop a new model, directly applicable to this class of solar cells, to determine which factors influence the device operation. Recently "bilayer" polymer based solar cells have been very successfully modelled by considering that at open-circuit there must be a balance between drift and diffusion currents in the device. ${ }^{20,21}$ Here, we develop an analogy to this theory applicable to the nanostructured solid-state dye-sensitized solar cell.

\section{A. Electric field distribution through the active layer}

First, we investigate how the electric field varies with distance throughout the film. It is close to impossible to microscopically measure the electrical potential through a cross section of a device, since it is too thin to achieve alignment of the microscope. However, we can make an in-plane nongated transistor type structure on a much larger length scale to the conventional device, and measure the surface potential as a function of distance between two electrodes. We have fabricated devices comprising of a nanostructured $\mathrm{TiO}_{2}$ film on glass, coated with a monolayer of dye molecules and infiltrated with Spiro-MeOTAD. Two gold electrodes were evaporated on top with a channel width of $100 \mu \mathrm{m}$. In Fig. 1(a) we show the surface potential near the middle of the channel, as a function of distance from the gold electrodes, as measured by a Kelvin probe microscope under ultrahigh vacuum with ambient lighting. Also presented in Fig. 1(b) is
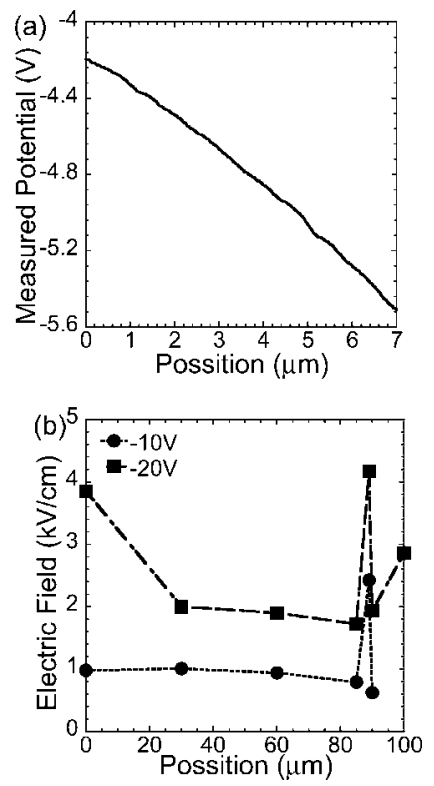

FIG. 1. (a) Surface potential in the middle of the channel of an in-plane device comprising of $\mathrm{TiO}_{2}$ nanoporous film/dye/SpiroMeOTAD with gold electrodes $100 \mu \mathrm{m}$ apart, and with $20 \mathrm{~V}$ potential difference between the two electrodes. The position of $0 \mu \mathrm{m}$ on this scale corresponds to approximately $60 \mu \mathrm{m}$ from the left electrode. (b) Electric field in the channel, calculated at various positions from the left electrode $(X=0 \mu \mathrm{m})$ as $\Delta V / \Delta X$, with a potential difference of $10 \mathrm{~V}$ and $20 \mathrm{~V}$ between the two electrodes. All these measurements were performed on a device fabricated with the Spiro-MeOTAD solution containing all the additives at the same concentrations used in the solar cell.

the electric field within the film at various positions across the channel, calculated from the gradient of the surface potential. Although the active layer is identical to that in the solar cell, we note that this is not the exact situation since we are only electrically contacting the hole transporter, no voltage can be generated from the device since it is symmetrical.

When no additives are added to the Spiro-MeOTAD we observe no contact resistance and a uniform drop in potential between the two electrodes. This is consistent with an electric field existing throughout the $100 \mu \mathrm{m}$ between the electrodes. Quite unexpectedly, when the hole-transporter has its full complement of additives ( $\mathrm{Li}$ salts, tertbutyl pyridine, and chemical p-dopant) the potential still drops uniformly between the electrodes. This implies that we really can and must consider both drift and diffusion currents in the solidstate dye-sensitized solar cell. We note that there is some microstructure in the potential, consistent with local redistribution of the ionic species.

\section{B. Charge density from current continuity}

For an initial simplification we consider the nanoporous film to be flattened out consisting of a layered structure of fluorene doped tin oxide (FTO) bottom electrode, $\mathrm{TiO}_{2}$, dye, Spiro-MeOTAD, and a gold top electrode. Neglecting space charge and ion screening effects, the voltage drop across the Spiro-MeOTAD is $V_{p}$ and the average electric field across 
this layer is $V_{p} / d_{p}$, where $d_{p}$ is the distance between the dye interface and the hole-collection electrode. At open circuit, the drift and diffusion currents must balance, thus the net current density is ${ }^{20}$

$$
J=q \mu_{p} p \frac{V_{p}}{d_{p}}+q D_{p} \frac{d p}{d x}=0,
$$

where $p, \mu_{p}$, and $D_{p}$ are the hole number density, mobility, and diffusion coefficient, respectively. The effective diffusion coefficient in the $\mathrm{TiO}_{2}$ is known to be dependent upon the electron density in the material and thus could be considered position dependent, and likewise for the hole diffusion coefficient and mobility in the Spiro-MeOTAD. However, we ignore this effect in order to obtain an analytical expression. Using a modified version of the Einstein relation, which takes into account the electronic disorder in the materials, $D / \mu=2 k T / q,{ }^{22-24}$ we obtain

$$
p(x)=p(0) \exp \left(-\frac{q V_{p}}{2 k T} \frac{x}{d_{p}}\right) .
$$

A similar expression has been previously derived and successfully applied to polymer based solar cells. ${ }^{20,21}$ Integrating Eq. (2) between the interface $(x=0)$ and the electrode $\left(x=d_{p}\right)$ gives the total number of charges per unit area of interface as

$$
p_{\text {int }}=p(0) 2 \frac{d_{p} k T}{q V_{p}} \quad \text { for } q V_{p} \gg k T .
$$

Above, we have simplified the system to consist of a flat layered structure. However, the interfacial surface area of the nanoporous film has a multiplicity factor of approximately 100 times per micron thickness larger than an equivalent flat interface. ${ }^{25}$ To obtain an expression for the total number of charges per unit area of film, $p_{\text {film }}$, we multiply Eq. (3) by a prefactor $100 \times L$, where $L$ is the film thickness in microns. Here we assume that the voltage drop between each part of the dye interface and the gold electrode is the same, and $d_{p}$ is now the "average" distance between the interface and the gold electrode.

There may be a higher charge density on one side of the film than on the other, since illumination is incident through one side. However, since the charge generation sites are distributed throughout the film we assume the charge density to be approximately uniform at open circuit, then the average hole density within the film, $p$, is $p_{\text {film }} / L$. This gives,

$$
p=p(0) 200 d_{p} \frac{k T}{q V_{p}} .
$$

Note that the prefactor 200 has units of $\mu \mathrm{m}^{-1}$ and $d_{p}$ is in microns.

\section{Charge density from the steady state rate equation}

Above we have formulated an expression for the charge density assuming current continuity. In the steady state at open circuit, we can also express a balance between charge generation and recombination as

$$
\frac{d p}{d t}=-p k_{\mathrm{rec}}+G=0
$$

where $p$ is the average charge density, $k_{\text {rec }}$ is the charge recombination rate constant, and $G$ is the average charge generation rate per unit volume.

There is a notion that the charge recombination in dyesensitized solar cells is "transport limited," governed by a trapping/detrapping process of electrons in the $\mathrm{TiO}_{2} \cdot 9,26,27$ We have recently investigated the recombination mechanism in the solid-state dye-sensitized solar cell and demonstrated that transport limited recombination is unlikely to be applicable to this system. ${ }^{28}$ We show that the recombination can be described by a "thermally assisted tunnelling" mechanism. It appears to be almost entirely governed by the tunnelling of electrons from the $\mathrm{TiO}_{2}$, through the dye molecules, to recombine directly with the holes in the SpiroMeOTAD. When the position of the Fermi level in the $\mathrm{TiO}_{2}$ is low there is an additional thermal activation $(\Delta E)$ to the charge recombination. The thermal activation is likely to arise from preferential recombination from higher energy sites. The charge recombination rate constant takes the following form: ${ }^{28}$

$$
k_{\mathrm{rec}}=k_{0} I^{\beta} \exp (-2 \gamma r) \exp \left(-\frac{\Delta E}{k T}\right),
$$

where $k_{0}$ is a recombination constant, $I^{\beta}$ describes the intensity dependence of the recombination process (for pure bimolecular recombination we expect $\beta \sim 0.5$ ), gamma is the inverse localization radius of the electron in the tunnelling direction (through the dye molecule), $r$ is the distance through which the electron must tunnel to recombine with the hole, and $\Delta E$ is the thermal activation energy which assists in the recombination process, $\Delta E \geqslant 0$.

Setting $k_{\text {rec }}$ from Eq. (6) into Eq. (5), and equating this to Eq. (4) gives

$$
p(0)=\frac{G}{200 k_{0} I^{\beta} d_{p}} \frac{q V_{p}}{k T} \exp (2 \gamma r) \exp \left(\frac{\Delta E}{k T}\right) .
$$

At $x=d_{p}$, Eq. (2) can be rewritten as $\ln [p(0)]-\ln \left[p\left(d_{p}\right)\right]$ $=q V / 2 k T$. Replacing $p(0)$ from this expression with Eq. (7) leads to our expression for the voltage drop across the SpiroMeOTAD layer as

$$
V_{p}=\frac{\Delta E}{q}+\frac{k T}{q}\left[2 \gamma r-\ln \left(\frac{200 k_{0} I^{\beta} d_{p}}{G} p\left(d_{p}\right)\right)\right] \text { for } q V \gg k T .
$$

\section{Intensity and thickness dependence of the charge generation rate}

Equation (8) gives a thickness dependence of the voltage drop across the Spiro-MeOTAD layer as $-\ln \left(d_{p}\right)$. However, the average charge generation rate is also thickness dependent since the average optical power density within the film will reduce with increasing thickness due to "preabsorption" of light. We can consider $G$, the average charge generation rate density, as 


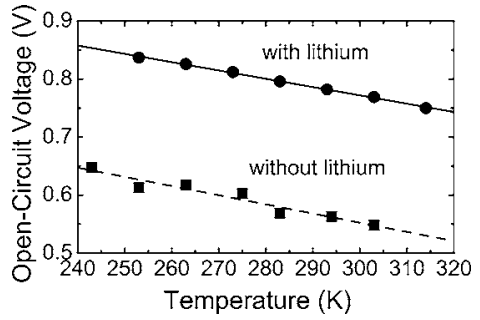

FIG. 2. Open-circuit voltage vs temperature for $1.8 \mu \mathrm{m}$ thick solar cells, with Li (solid line, circles) and without Li (dashed line, squares). The symbols are the data points and the lines are linear fits to the data.

$$
G=g\left(\frac{1-\exp (-\alpha L)}{L}\right) I,
$$

where $g$ is the charge generation efficiency from absorbed photons, $I$ is the number of incident photons per unit area per unit time, and $\alpha$ is the absorption coefficient of the sensitized film of thickness $L$. We are neglecting reflected light off the back electrode and subsequent interference effects. Setting this expression for $G$ into Eq. (8) gives

$$
V_{p}=\frac{\Delta E}{q}+\frac{k T}{q}\left[2 \gamma r-\ln \left(\frac{200 k_{0} p\left(d_{p}\right) d_{p} L}{I^{(1-\beta)} g\left(1-e^{-\alpha L}\right)}\right)\right] .
$$

We can derive a similar expression for the voltage drop across the $\mathrm{TiO}_{2}$ layer $\left(V_{n}\right)$ with the only difference being that $d_{p}$ is replaced with $d_{n}$, the average distance between the charge generation interface and the FTO electrode. The open-circuit voltage is then $V_{\mathrm{oc}}=V_{p}+V_{n}+\phi_{B} / q+\Delta \phi / q$, where $\phi_{B}$ is the built-in potential dictated by the work function differences between the two electrodes, and $\Delta \phi$ is a potential shift at the dye interface which may occur if a surface dipole is present, giving,

$$
\begin{aligned}
V_{\mathrm{oc}}= & \frac{\phi_{B}+\Delta \phi+2 \Delta E}{q}+\frac{k T}{q} \\
& \times\left[4 \gamma r-\ln \left(\frac{40000 k_{0}^{2} p\left(d_{p}\right) n\left(d_{n}\right) d_{p} d_{n} L^{2}}{I^{2(1-\beta)} g^{2}\left(1-e^{-\alpha L}\right)^{2}}\right)\right] .
\end{aligned}
$$

We note that if Fermi level pinning occurs or dipole moments exist at either electrode then the value for the built-in potential may differ significantly from the difference between the work functions of the two electrodes. ${ }^{29}$ Furthermore, it is unlikely to be greater than the difference between the $\mathrm{TiO}_{2}$ conduction band and the highest occupied molecular orbital (HOMO) energy level of the Spiro-MeOTAD.

\section{E. Temperature dependence of the $V_{\text {oc }}$}

Figure 2 shows the open-circuit voltage versus temperature for solid-state cells with and without the addition of Li ions ( $\mathrm{Li}$ salts are added as they improve the device performance characteristics $\left.{ }^{8,16}\right)$. As predicted we have a linear relationship, with a negative gradient for both devices. For the device with no Li ions the $y$-axis intercept at zero Kelvin is approximately $1 \mathrm{~V}$. If we assume that the maximum built in potential is $0.6 \mathrm{eV}$, calculated as the difference between the
$\mathrm{TiO}_{2}$ conduction band and the Spiro-MeOTAD HOMO energy level. Then we appear to have a contribution of $0.4 \mathrm{eV}$ from surface dipoles and the thermal activation energy to charge recombination. Using a macroscopic Kelvin probe we have independently measured the surface potential of flat $\mathrm{TiO}_{2}$ films with and without surface coverage of the dye molecules. We observe a shift of approximately $150 \mathrm{meV}$ when the dye molecules are present on the surface $(4.8 \mathrm{~V}$ for clean $\mathrm{TiO}_{2}$ as compared with $4.65 \mathrm{~V}$ for dye coated $\mathrm{TiO}_{2}$ in comparison with a gold reference electrode measured at $5.1 \mathrm{~V})$. This dipole moment, permanently induced by the dye molecules, is expected since the LUMO energy levels are predominantly close to the $\mathrm{TiO}_{2}$ and the HOMO energy levels exist on the NCS groups pointing away from the $\mathrm{TiO}_{2}$. This implies that we have an activation energy to recombination $(\Delta E)$ of $125 \mathrm{meV}$, reasonably consistent with what we have estimated from transient open-circuit voltage decay measurements taken over a range of temperature. ${ }^{28}$ Assuming the same built-in potential and activation energy for the $\mathrm{Li}$ doped device, the addition of $\mathrm{Li}$ ions appears to have induced further permanent surface dipoles resulting in a potential shift of $0.2 \mathrm{eV}$. We note that the maximum opencircuit voltage (at zero $\mathrm{K}$ ) of $1.2 \mathrm{~V}$ is identical to that determined by Kron et al. for the liquid cell, ${ }^{11}$ demonstrating, contrary to their findings, that the solid-state device can indeed have an open-circuit voltage as high as, if not higher than, the liquid cell.

There are two oppositely varying temperature dependent factors which affect the open-circuit voltage. First the diffusion of charge will increase more rapidly with temperature than the charge mobility. Thus, in order to balance drift and diffusion currents at open circuit a higher voltage must be applied across the device as the temperature increases. In the opposite manner, as the temperature rises the charge recombination rate increases. Assuming that the charge generation rate remains constant, then for steady state to be achieved the charge density within the device will have to fall, reducing the open-circuit voltage. The latter appears to be the dominant effect. Although we have not considered it here, we also note that we have previously observed a slight reduction in the dipole offset between the $\mathrm{TiO}_{2}$ and the Spiro-MeOTAD as the temperature is raised. ${ }^{28}$ This will also contribute to the reduction in open-circuit voltage with increasing temperature.

\section{F. Thickness dependence of the $V_{\text {oc }}$}

The relationship between $d_{p}, d_{n}$, and $L$ is not straightforward. Clearly there will be a reduction in the light absorption throughout the thickness of the film, implying that $d_{n}$ is less strongly dependent on thickness than $d_{p}$ (light is incident through the electron collecting electrode). Also, interference effects from reflected light off the gold "back" electrode will further complicate the situation. However, as a simple approximation to demonstrate the validity of our model, we take $d_{p}=d_{n}=L / 2$. Figure 3 shows the open-circuit voltage plotted against $\ln \left\{L^{4} /[1-\exp (-\alpha \mathrm{L})]^{2}\right\}$ for devices illuminated by simulated sun light at 10,53 , and $100 \mathrm{~mW} \mathrm{~cm}^{-2}$. We have estimated $\alpha$, the absorption coefficient, to be 


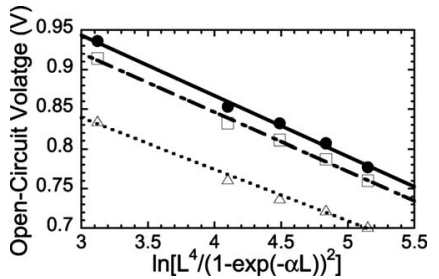

FIG. 3. Open-circuit voltage vs $\ln \left\{L^{4} /[1-\exp (-\alpha L)]^{2}\right\}$ for devices measured under simulated AM 1.5 illumination at $10 \mathrm{~mW} \mathrm{~cm}^{-2}$ (open-triangles, dotted line), $53 \mathrm{~mW} \mathrm{~cm}^{-2}$ (open squares, dotted-dashed line), and $100 \mathrm{~mW} \mathrm{~cm}^{-2}$ (solid circles, solid line). The lines are linear fits to the data.

$0.3 \mu \mathrm{m}^{-1}$ from UV-vis transmission spectroscopy on sensitized $\mathrm{TiO}_{2}$ films. We observe a straight line at all intensities and have a similar gradient for all curves, demonstrating that this function correctly describes the relationship between voltage and thickness for these devices. However, we obtain a slightly larger value for the gradient than expected, $2.5 \times k T / q$. This may be due to the estimation of $d_{p}=d_{n}=L / 2$. A further contribution is likely to arise from incomplete filling of the nanoporous film with SpiroMeOTAD. This will be worse for the thicker films and is likely to give a steeper reduction of the open-circuit voltage with thickness than is expected.

There exist two physical origins of the thickness dependence of the open-circuit voltage. First, the average optical power density within the film reduces as the film becomes thicker, due to "preabsorption." This results in a reduction of the average charge density at the light absorbing interface and thus of the open-circuit voltage. The second origin is less intuitive, it derives from the charge generation sites, and more importantly the charge recombination sites, being distributed throughout the film. Even with a flat and constant optical power density the voltage would still reduce with thickness. We interpret this as implying that as the film becomes thicker the charges have more chance of recombining before influencing the potential at the electrodes. It is important to note that Eq. (4) would have no thickness dependence if the device consisted of a flat layered structure and the thickness dependence arises from the multiplicity factor $100 \times L$ when converting the equation for the flat junction into the nanoporous structure. This is likely to be the manifestation of the charge diffusion length being comparable with the film thickness.

\section{G. Intensity dependence of the $V_{o c}$}

Figure 4(a) shows the open-circuit voltage versus incident illumination intensity at room temperature for devices of various thicknesses. We find that the gradient is approximately $1.7 \mathrm{kT} / \mathrm{q}$ for all devices. Figure 4(b) shows the opencircuit voltage versus incident illumination intensity at 244 and $303 \mathrm{~K}$ for a device $2 \mu \mathrm{m}$ thick. The gradients are similar to before at $1.65 \mathrm{kT} / \mathrm{q}$ and $1.6 \mathrm{kT} / \mathrm{q}$, respectively, demonstrating the applicability of Eq. (11) to the intensity dependence of the open-circuit voltage. From these values we estimate $\beta$, the degree to which the charge lifetime varies with
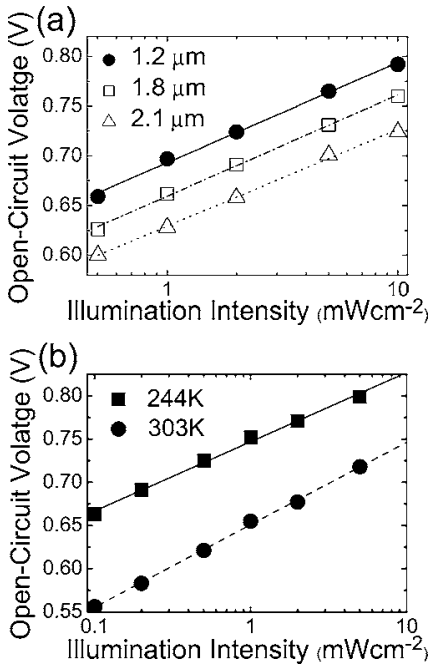

FIG. 4. (a) Open-circuit voltage vs illumination intensity for devices with a thickness of 1.2, 1.8, and $2.1 \mu \mathrm{m}$ (see legend). (b) Open-circuit voltage versus illumination intensity for a device $2 \mu \mathrm{m}$ thick at $244 \mathrm{~K}$ and $303 \mathrm{~K}$. The lines are logarithmic fits to the data.

intensity, to be approximately 0.2 . We have previously performed transient open-circuit voltage decay measurements and independently determined the charge recombination rate constant as a function of illumination intensity. We find it varies as approximately $I^{0.5},{ }^{28}$ which is comparable to, though a little larger than, the value estimated here. We note that a variation of $I^{0.5}$ implies that we have predominantly bimolecular recombination, similar to that in an all polymer solar cell. ${ }^{20}$

The physical origin of the intensity dependence of the open-circuit voltage is clear and well understood for other systems. ${ }^{20,21,30} \mathrm{An}$ increased charge generation rate results in an increased chemical potential within the device and thus an increase in the open-circuit voltage. However, the reduced charge lifetime, reduces the chemical potential within the device and acts as to reduce the open-circuit voltage. The observed behavior is due to the charge generation rate increasing more strongly with intensity than the reduction of the charge lifetime.

Preliminary results for liquid electrolyte cells show similar trends, though there are subtle differences which are currently under investigation.

\section{CONCLUSION}

In conclusion, by considering a balance between drift and diffusion currents and between charge generation and recombination we have formulated an expression for the temperature, thickness, and light intensity dependence of the opencircuit voltage for solid-state dye-sensitized solar cells. The derived formula accurately follows the experimentally observed trends, and proves to be exceptionally useful for deducing the physical origin of the open-circuit voltage, especially concerning the subtle processes occurring at the dye interface. 


\section{ACKNOWLEDGMENTS}

This work was funded by the MOLYCELL European project (OFES 03.0681-1). The authors are very grateful to
Michel Schaer and Libero Zuppiroli from the Laboratory of Optoelectronics of Molecular Materials, EPFL, where most of the experiments were performed. The Kelvin probe microscope was funded by the EPSON Cambridge laboratory.
*Institut de Chimie Physique, École Polytechnique Fédérale de Lausanne, CH-1015 Lausanne, Switzerland; Electronic address: henry.snaith@epfl.ch

${ }^{1}$ U. Bach, D. Lupo, P. Comte, J. E. Moser, F. Weissortel, J. Salbeck, H. Spreitzer, and M. Gratzel, Nature (London) 395, 583 (1998).

${ }^{2}$ B. O'Regan and M. Grätzel, Nature (London) 353, 737 (1991).

${ }^{3}$ L. Schmidt-Mende, S. M. Zakeeruddin, and M. Grätzel, Appl. Phys. Lett. 86, 013504 (2005).

${ }^{4}$ P. Wang, B. Wenger, R. Humphry-Baker, J. E. Moser, J. Teuscher, W. Kantlehner, J. Mezger, E. V. Stoyanov, S. M. Zakeeruddin, and M. Grätzel, J. Am. Chem. Soc. 127, 6850 (2005).

${ }^{5}$ P. Ravirajan, D. D. C. Bradley, J. Nelson, S. A. Haque, J. R. Durrant, H. J. P. Smit, and J. M. Kroon, Appl. Phys. Lett. 86, 143101 (2005).

${ }^{6}$ P. A. van Hal, M. M. Wienk, J. M. Kroon, and R. A. J. Janssen, J. Mater. Chem. 13, 1054 (2003).

${ }^{7}$ S. A. Haque, T. Park, C. Xu, S. Koops, N. Schulte, R. J. Potter, A. B. Holmes, and J. R. Durrant, Adv. Funct. Mater. 14, 435 (2004).

${ }^{8}$ J. Kruger, R. Plass, L. Cevey, M. Piccirelli, M. Grätzel, and U. Bach, Appl. Phys. Lett. 79, 2085 (2001).

${ }^{9}$ J. Nelson, S. A. Haque, D. R. Klug, and J. R. Durrant, Phys. Rev. B 63, 205321 (2001).

${ }^{10}$ F. Pichot and B. A. Gregg, J. Phys. Chem. B 104, 6 (2000).

${ }^{11}$ G. Kron, T. Egerter, J. H. Werner, and U. Rau, J. Phys. Chem. B 107, 3556 (2003).

${ }^{12}$ I. Mora-Sero, T. Dittrich, A. Belaidi, G. Garcia-Belmonte, and J. Bisquert, J. Phys. Chem. B 109, 14932 (2005).

${ }^{13}$ L. Kavan and M. Gratzel, Electrochim. Acta 40, 643 (1995).

${ }^{14}$ H. J. Snaith and M. Grätzel, Adv. Mater. (Weinheim, Ger.) (to be published).

${ }^{15}$ C. J. Barbe, F. Arendse, P. Comte, M. Jirousek, F. Lenzmann, V. Shklover, and M. Gratzel, J. Am. Ceram. Soc. 80, 3157 (1997).

${ }^{16}$ H. J. Snaith, S. M. Zakeeruddin, L. Schmidt-Mende, C. Klein, and M. Grätzel, Angew. Chem., Int. Ed. 44, 6413 (2005).

${ }^{17}$ D. Kuang, C. Klein, H. J. Snaith, R. Humphry-Baker, J.-E. Moser, S. M. Zakeeruddin, and M. Grätzel, Nano Lett. 6, 769
(2006).

${ }^{18}$ L. Bürgi, H. Sirringhaus, and R. H. Friend, Appl. Phys. Lett. 80, 2913 (2002).

${ }^{19}$ B. A. Gregg, J. Phys. Chem. B 107, 4688 (2003).

${ }^{20}$ C. M. Ramsdale, J. A. Barker, A. C. Arias, J. D. MacKenzie, R. H. Friend, and N. C. Greenham, J. Appl. Phys. 92, 4266 (2002).

${ }^{21}$ J. A. Barker, C. M. Ramsdale, and N. C. Greenham, Phys. Rev. B 67, 075205 (2003).

${ }^{22}$ It is well understood that the Einstein relation does not hold for all disordered semiconductors deviating from the classical value of $k T / q$ when there is a tail to the DOS below the conduction band. In this instance a generalized form of the Einstein relation should be employed which takes into account the DOS function and the charge concentration. If the charge concentration is low, which is likely to be the case under photovoltaic operation, then the deviation is small. Furthermore, if the "trap states" are distributed exponentially beneath the conduction band (charge transport level for organics), as is likely to be the case for $\mathrm{TiO}_{2}$ and most disordered organic semiconductors, the deviation is a constant factor, and will be approximately 2 for the materials used herein. See the following two references.

${ }^{23}$ Y. Roichman and N. Tessler, Appl. Phys. Lett. 80, 1948 (2002).

${ }^{24}$ J. van de Lagemaat, N. Kopidakis, N. R. Neale, and A. J. Frank, Phys. Rev. B 71, 035304 (2005).

${ }^{25}$ M. Grätzel, Nature (London) 414, 338 (2001).

${ }^{26}$ N. Kopidakis, K. D. Benkstein, J. van de Lagemaat, and A. J. Frank, J. Phys. Chem. B 107, 11307 (2003).

${ }^{27}$ S. A. Haque, Y. Tachibana, R. L. Willis, J. E. Moser, M. Grätzel, D. R. Klug, and J. R. Durrant, J. Phys. Chem. B 104, 538 (2000).

${ }^{28}$ H. J. Snaith, S. M. Zakeeruddin, C. Klein, R. Humphry-Baker, and M. Grätzel (unpublished).

${ }^{29}$ C. J. Brabec, A. Cravino, A. D. Meissner, N. S. Sariciftci, T. Fromherz, M. T. Rispens, L. Sanchez, and J. C. Hummelen, Adv. Funct. Mater. 11, 374 (2001).

${ }^{30}$ L. J. A. Koster, V. D. Mihailetchi, R. Ramaker, and P. W. M. Blom, Appl. Phys. Lett. 86, 123509 (2005). 